\title{
Le Facebook des étudiants marocains. Territoire relationnel et territoire des possibles
}

Facebook of Moroccan Students. Relational Territory and Possible Territory Facebook de los estudiantes marroquíes. Territorio relacional y territorio de lo posible

\section{Sabrina Marchandise}

\section{(2) OpenEdition} Journals

\section{Édition électronique}

URL : https://journals.openedition.org/remi/7065

DOI : $10.4000 /$ remi.7065

ISSN : $1777-5418$

Éditeur

Université de Poitiers

Édition imprimée

Date de publication : 1 décembre 2014

Pagination : $31-48$

ISBN : 979-10-90426-23-8

ISSN : 0765-0752

Référence électronique

Sabrina Marchandise, «Le Facebook des étudiants marocains. Territoire relationnel et territoire des possibles ", Revue européenne des migrations internationales [En ligne], vol. 30 - n³ et 4 | 2014, mis en ligne le 01 décembre 2017, consulté le 15 avril 2022. URL : http://journals.openedition.org/remi/7065 DOI : https://doi.org/10.4000/remi.7065 


\section{Le Facebook des étudiants marocains. Territoire relationnel et territoire des possibles}

\section{Sabrina Marchandise ${ }^{1}$}

Si la géographie s'est largement renouvelée sur l'analyse des espaces de circulations et des territorialités mouvantes dans la migration, nous proposons ici un positionnement théorique faisant le lien entre les migrations, les réseaux de sociabilités et les pratiques web. Nous voulons rendre compte des transformations du rapport des migrants à l'espace et aux liens dans un contexte d'une société hypermobile où l'environnement est augmenté par I'utilisation des technologies de l'information et de la communication (TIC). L'analyse des migrations a ainsi évolué de l'immigré déraciné (" la double absence ", Sayad, 1999), au migrant circulant, jusqu'au migrant " connecté " (Diminescu, 2005) et à " la double présence " (Dufoix, 2003). Il est ainsi de moins en moins possible de penser le lien social en dehors des TIC. Cette approche s'insère au cœur des problématiques étudiées par les humanités digitales. Pluridisciplinaire, ce mouvement émergent est à l'intersection des sciences sociales et des technologies numériques.

Les migrants " connectés " gardent toujours le contact avec le pays d'origine, leur réseau social d'origine, mais peuvent élargir leurs relations en dehors de leur groupe d'appartenance. L'approche complémentaire entre les constructions transnationales et les communautés numériques permet alors une précision des pratiques et réseaux jusqu'à poser la question des impacts spatiaux (Proulx, 2008). Cet article questionne d'abord la place des pratiques du web dans les réseaux sociaux des étudiants marocains, la structuration et l'organisation de ces réseaux ainsi que la manière dont ils s'inscrivent dans l'espace. La connectivité transforme-t-elle le rapport à l'espace ? L'objectif est de déplacer la problématique de la mobilité internationale des étudiants en faisant le lien entre la dimension migratoire de la mobilité et les nouvelles technologies par une approche des réseaux sociaux de la mobilité. De nouvelles formes de territorialisation se développent alors à partir des réseaux au sein d'espaces discontinus et pourtant connectés dans la mobilité. II s'agit alors de voir en quoi les mobilités contribuent à l'émergence de nouveaux rapports à l'espace, et en quoi la spatialisation des réseaux sociaux est révélatrice de ces rapports.

1 Post-doctorante en géographie, Université Toulouse Jean Jaurès, LISST (UMR 5193), Maison de la recherche, 5 allée Antonio-Machado, 31058 Toulouse cedex 9 ; sabrina. marchandise@gmail.com 
Les mobilités étudiantes ne sont pas nouvelles, mais soulèvent des questions inédites dans le champ de recherche des migrations. Nous constatons, par ailleurs, que les mobilités étudiantes internationales sont rarement abordées comme construction migratoire spécifique. Dans le cadre de mobilités particulières (provisoires, passagères, éphémères), nous supposons la capacité des étudiants à mobiliser des réseaux multiples et élargis, sans exclure une solidité des réseaux inscrits dans la durée et le rapport à l'origine.

La démarche est de présenter le phénomène des mobilités étudiantes sous l'angle du point d'aboutissement des étudiants pour saisir l'ensemble des réseaux de la mobilité. Ainsi, délibérément, le contexte choisi est celui de l'espace d'accueil. L'intérêt de ce positionnement est de comprendre les réseaux de mobilité des étudiants marocains depuis l'étranger, c'est-à-dire de savoir qui ils sont, d'où et comment ils viennent, ce qu'ils font et ce qu'ils projettent.

Le choix de la France s'est imposé par les statistiques puisque la France reste la première destination des étudiants marocains en mobilité internationale et les étudiants marocains sont la première population étudiante étrangère en France. Ce choix est également motivé en fonction des critères historiques, de la connaissance d'une certaine élite marocaine formée en France. La définition des terrains est basée sur des critères statistiques relatifs à la présence des étudiants marocains dans les universités françaises. Les terrains sont entendus comme le lieu privilégié des observations et des enquêtes parmi les différentes destinations des étudiants marocains. Les statistiques nous amènent sur deux terrains : Montpellier et Lille, deuxième et troisième villes d'accueil des étudiants marocains.

L'investigation du web social des étudiants marocains fait suite aux entretiens réalisés auprès de soixante-dix-sept étudiants en France ${ }^{2}$. L'enquête permet $d^{\prime}$ avancer que leurs pratiques d'Internet sont principalement orientées vers le web social, particulièrement le réseau social Facebook ${ }^{3}$. La démarche impose des questionnements méthodologiques et théoriques autour de la composition des réseaux du web et de leur évolution dans le temps en corrélation avec les temps de mobilité. Pour répondre aux nombreux questionnements que posent les pratiques du web social, nous avons mobilisé les graphes de visualisation des réseaux à partir desquels les représentations et les pratiques de l'espace sont interrogées au regard des temporalités de la migration. Cette approche par les réseaux sociaux du web ouvre deux clés d'analyse importantes : une représentation objective de la territorialité migrante par son maillage social, temporel et spatial ; une exploration de l'espace tel qu'il est perçu par les migrants eux-mêmes.

Après l'identification des liens par les graphes, il convient de revenir sur les temporalités de la mobilité étudiante, du temps quotidien, aux étapes de la mobilité jusqu'à l'évolution des réseaux. En effet, l'approche par les temporalités

\footnotetext{
2 Dans le cadre d'une recherche doctorale, soixante-dix-sept entretiens biographiques ont été réalisés avec des étudiants marocains à Lille et à Montpellier pour l'analyse des trajectoires, des sociabilités, des représentations et des pratiques.
}

3 La moyenne de temps quotidien passé sur Facebook est entre trente minutes et cinq heures (en utilisation active), en sachant que la grande majorité des étudiants sont toujours connectés via le téléphone portable ou l'écran d'ordinateur (utilisation passive). 
des réseaux sociaux questionne le rapport à l'espace et, par conséquent, la définition des territoires. Nous interrogeons ensuite le territoire dans une acception relationnelle, c'est-à-dire dont les fondements seraient les relations sociales construites et entretenues dans le mouvement. Enfin, les résultats permettent de discuter le concept de territoire des possibles pour les étudiants en mobilité internationale. II se définit à partir des relations établies et/ou entretenues à distance et prend sens par une idée de projection envisageable. Les pratiques web incitent également à la révision du concept d'imaginaire migratoire développé par Simon (2008). Dans la mesure où la migration évolue avec les nouvelles technologies, la construction d'un imaginaire n'est plus un fantasme, mais est construit dans le lien via l'opérateur technique qu'est l'ordinateur. Ainsi, le discours relatif à l'imaginaire de la migration se déplace en fonction des outils et des technologies modifiant l'entretien des liens.

\section{Le Facebook des étudiants marocains en mobilité internationale : des réseaux structurés, dynamiques et inscrits dans l'espace}

L'exploration du web n'était pas particulièrement prévue en début de recherche, mais s'est imposée suite aux premiers entretiens. Les questions faisant référence aux moyens de garder le contact avec le pays d'origine, avec la famille et les amis ont fait émerger l'usage fréquent d'Internet et particulièrement des réseaux sociaux sur le web, avec en premier lieu Facebook. Ce sont donc les premiers éléments de terrain qui ont entraîné une vraie réflexion théorique et méthodologique non prévue initialement. La démarche de terrain est donc inductive et s'est faite dans un va-et-vient avec le processus de théorisation sur le traitement géographique des données du web. La méthode n'est pas stabilisée, elle a été construite en marchant. Cette recherche est expérimentale et mène une réflexion sur la manière dont la géographie peut et doit s'emparer de la traçabilité du social sur le web et des impacts spatiaux. Nous faisons l'hypothèse de réseaux élargis construits dans des temporalités très distinctes : réseau des amis marocains d'avant la mobilité étudiante internationale, réseau familial, réseau élargi construit pendant la mobilité. La structuration de ces réseaux serait en partie basée sur l'origine géographique, mais également sur d'autres éléments liés à la mobilité internationale des étudiants.

\section{L'identification des liens par les graphes}

Parmi les étudiants du corpus d'entretiens biographiques, vingt d'entre eux ont participé au protocole mis en place pour l'étude des réseaux Facebook dans le cadre du programme "e-Diasporas $" 4$. Afin de respecter le consentement libre et éclairé, ainsi que la possibilité de se retirer à tout moment de l'étude, chacun des étudiants s'est rendu sur une page web spécialement créée (http:// fb.tactiques.org/index.php) et s'est inscrit directement depuis son ordinateur (Figure 1).

\footnotetext{
4 Projet ANR « e-Diasporas Atlas » coordonné par la FMSH - Programme de recherche TIC-Migrations - et sous la responsabilité de Dana Diminescu. Pour l'analyse des réseaux Facebook, la dimension exploratoire a guidé la démarche méthodologique. Ainsi, pour une question de faisabilité, vingt étudiants ont participé au protocole, sans sélection particulière, mais avec des profils variés.
} 
Figure 1 : Capture d'écran de la page de présentation du protocole Facebook

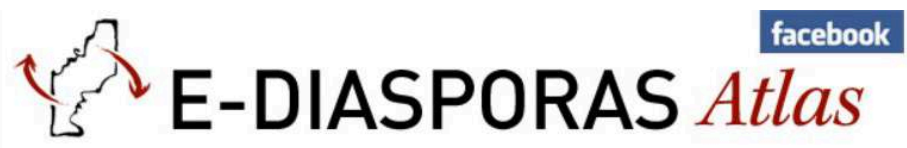

Dans le cadre de la recherche doctorale de Sabrina Marchandise, cette application va permettre de suivre l'évolution de votre réseau Facebook.

Pour cela, vous avez besoin de vous identifier grâce à votre compte facebook à l'aide du boutton de connexion ci-dessous.

Toutes les données recueillies seront confidentielles et traitées anonyemement. À tout moment, si vous souhaitez vous retirer de cette recherche merci de nous le signaler et nous ferons le nécessaire.

ff $\log$ In

Pour toutes questions ou informations, n'hésitez pas à nous contactez:

Le programme Tic-Migrations : tic-migrations[at]msh-paris.fr Sabrina Marchandise : sabrina.marchandise[at]gmail.com

Source : http://fb.tactiques.org/index.php

Dans un premier temps, à partir des données recueillies, I'utilisation de graphes de liens ${ }^{5}$ non spatialisés permet de représenter les sociabilités des étudiants sur Facebook (Figure 2). Sur les graphes de réseaux personnels, les étudiants dont le réseau est représenté n'apparaissent pas puisqu'ils sont par définition reliés à tous leurs amis. Les " amis " Facebook sont matérialisés par un point (un nœud du réseau) et les liens entre les amis par des traits, ce qui met en évidence qui est ami avec qui dans ce réseau. Chaque relation est donc représentée par un trait, mais celui-ci ne rend pas compte de l'épaisseur des relations ni de leurs contenus.

Dans un second temps, la compréhension du graphe a été rendue possible à partir des entretiens de commentaires du graphe par les étudiants eux-mêmes. Le graphe est mobilisé comme instrument privilégié pour que les enquêtés resituent les réseaux qui apparaissent (Figure 3). Après un premier entretien biographique déjà réalisé, il est intéressant de voir comment les discours évoluent dans la présentation des réseaux. La position réflexive des enquêtés permet de donner sens au graphe, de saisir une logique de représentation des réseaux non décryptable pour le chercheur seul et d'ancrer la représentation des liens dans le vécu des personnes. La mise en mots de cette confrontation au graphe permet de resituer I'historique de construction des réseaux et d'en identifier les différents clusters ou sphères. À partir du graphe, nous pouvons mettre en avant le parcours de l'étudiant depuis le pays d'origine jusqu'à aujourd'hui et avoir une connaissance des réseaux que l'étudiant entretient, crée ou laisse derrière lui. Les individus combinent de multiples appartenances que I'on retrouve sur la représentation de leur réseau Facebook.

5 Les données sont récupérées par I'API Facebook. Ce travail a été réalisé dans le cadre du programme " e-Diasporas". 
Figure 2: Graphe du réseau Facebook d'une étudiante marocaine à Montpellier

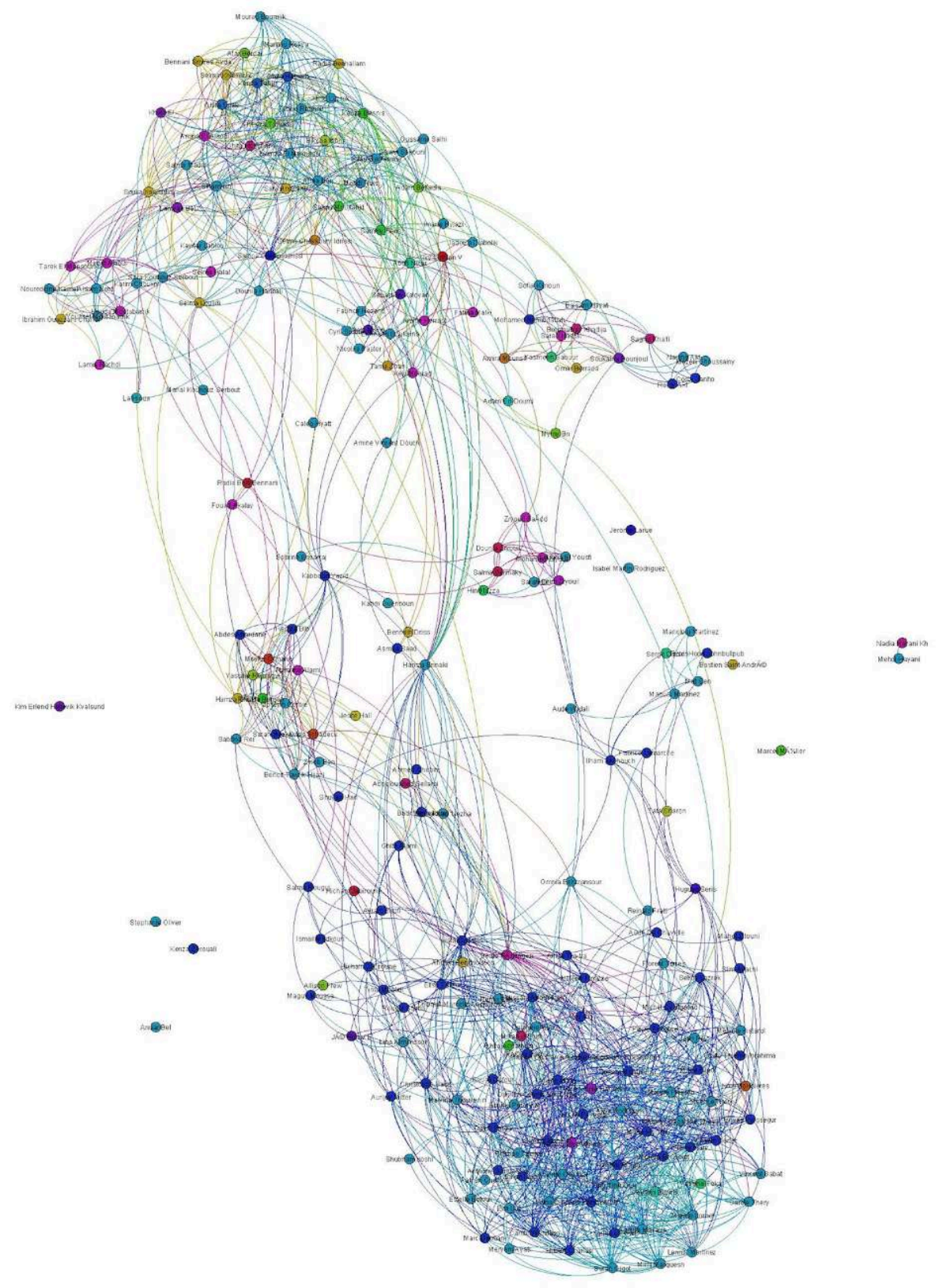

Source : Données API Facebook mises en graphe avec le logiciel Gephi. 
Figure 3 : Graphe commenté par l'étudiante de son réseau Facebook

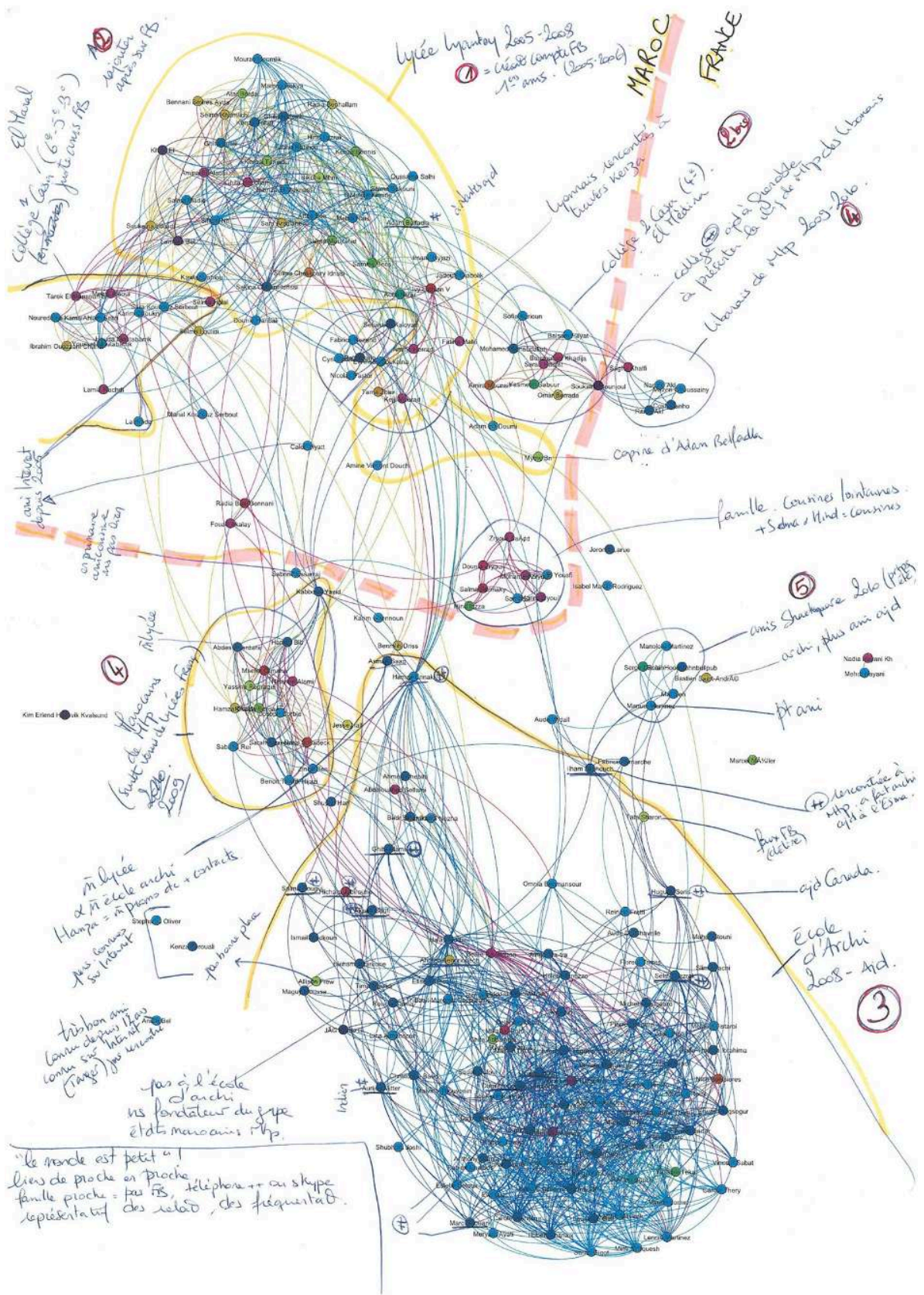

Source : Données API Facebook mises en graphe avec le logiciel Gephi. Entretien de commentaires du graphe par l'étudiante. 
Les principaux résultats issus de cette méthode sont l'identification de l'origine de la formation des clusters et la compréhension de leur organisation, tant spatiale que temporelle.

\section{La formation des clusters}

Au début des entretiens de commentaires des graphes, les étudiants sont surpris et s'attachent à comprendre la logique du graphe. Ils sont, dans un premier temps, souvent attirés par les clusters plus importants. Ils tentent $d^{\prime}$ abord d'avoir une vision d'ensemble en identifiant des noms connus au sein de chaque cluster. Très vite, le graphe prend sens. Les étudiants repèrent les différents groupes d'amis. Ils expliquent pourquoi et comment existent les clusters. Nous retrouvons les clusters collège, lycée, famille (plus limité en nombre), études supérieures et rencontres dans la ville d'études, associations, etc. En général, les étudiants resituent tout de suite les différentes personnes déjà mentionnées lors du premier entretien puis, à partir de là, expliquent les liens avec les autres personnes. L'importance des clusters en volume n'est pas toujours proportionnelle à l'importance affective quotidienne.

\section{L'organisation des clusters}

Les étudiants rendent très vite compte de la séparation des clusters selon l'origine géographique des connaissances et non pas selon leur localisation actuelle. En effet, on retrouve toujours un cluster correspondant aux amis du lycée et qui peuvent aujourd'hui être dispersés géographiquement. C'est le cas des amis de M., étudiante à Montpellier :

"On voit bien un groupe avec tous les amis du lycée français de Tanger, mais aujourd'hui la plupart sont en France, dans des villes différentes, et certains sont dans d'autres pays".

On retrouve toujours une "frontière " géographique France/Maroc dans le sens où les groupes d'amis rencontrés au Maroc ou en France sont nettement distincts. Cette frontière n'est que virtuelle puisque la position des individus sur le graphe ne correspond pas à leur localisation géographique. Les liens sont plus ou moins développés entre les clusters au Maroc et en France, souvent selon la mobilité internationale des amis du lycée. Dans le cas de M. qui a fait le lycée français de Casablanca, beaucoup de ses amis sont partis en France après le baccalauréat et ont donc fait le lien avec d'autres groupes d'amis rencontrés en France.

Le web social des étudiants marocains représente finalement un espace social, comme l'espace social réel, avec la particularité de contracter l'ensemble des liens et des connaissances acquises au cours des mobilités et des temporalités de la vie.

" Je me sers de Facebook pour tous les amis, avec les amis ici et au Maroc, d'ailleurs c'est un avantage parce qu'on n'appelle pas forcément les amis au Maroc alors Facebook c'est une facilité pour garder le lien, je peux leur parler tous les jours, ce qui aurait été impossible il y a encore quelques années. Ça permet de garder le contact avec ceux à Paris, à Nice, aux États-Unis et je sais que je pourrai garder le contact avec ceux que je vais rencontrer dans les futurs voyages. Donc au final, c'est vraiment une toile qui se 
tisse entre nous tous. Facebook c'est une ouverture vers le monde et en même temps ça me rattache au Maroc " ( $\mathrm{H}$. , étudiant à Montpellier).

Le réseau Facebook des étudiants marocains traduit une vision du monde partagée entre l'ouverture et I'ancrage au Maroc. La première description spatiale du réseau suite aux entretiens fait référence à des réseaux de connaissance très ancrés au Maroc et dans la ville d'études, à une conservation des liens forts à distance, à un éclatement géographique des liens traduisant les mobilités internationales et à une majorité de Marocains au sein du réseau (mais pas tous localisés au Maroc : homophilie d'éducation, d'origine sociale, d'études, etc.).

Ces réseaux permettent la mise en évidence des logiques de continuité et des logiques d'élargissement des réseaux au fur et à mesure des étapes de mobilité. Les clusters correspondent ainsi aux temporalités de la vie des étudiants qui apparaissent sur le graphe. Celles-ci sont différentes des temporalités d'ajout des amis sur Facebook, puisque la création du compte date soit du lycée, soit des études supérieures. Nous retrouvons ainsi les différentes étapes de la vie des étudiants.

\section{Le web social révélateur des temporalités en migration}

Certains auteurs (Di Méo, 1998 ; Marié, 1982 ; Raffestin, 1982) ont établi le fait que le territoire ne pouvait se construire et exister qu'avec " l'épaisseur du temps ". Très représentatif de ce point de vue, Marié (1982) estime que "l'espace a besoin de l'épaisseur du temps, de répétitions silencieuses, de maturations lentes, du travail de l'imaginaire social et de la norme pour exister comme territoire ". À l'inverse, nous nous inscrivons ici plutôt dans une approche des territorialités des temps courts, du quotidien et qui ne dure pas. À partir des réseaux Facebook des étudiants marocains, plusieurs niveaux de temporalités sont identifiables. Tout d'abord comment penser le temps quotidien qui rythme la mise en lien ? Nous abordons ce premier aspect des temporalités à partir des rythmes de l'échange et de la dynamique des relations sociales. Ensuite, ce sont les temporalités au regard du parcours de mobilité internationale, dans un temps plus long, celui des étapes de mobilité. Enfin, les temporalités se révèlent être indispensables pour questionner l'enjeu de l'évolution des réseaux.

\section{Temps quotidien : les rythmes de l'échange}

Le premier niveau de temporalité s'attarde sur les rythmes de l'échange et la dynamique des relations sociales. Au cours d'une période de vie en mobilité internationale, il est intéressant de saisir le rythme de la mise en relation : temps de l'absence, temps de la co-présence, temps de la rencontre et des interactions médiatisées à distance. Les étudiants ont souvent la particularité d'avoir un grand nombre de connaissances et un emploi du temps chargé (cours, travail personnel, sorties, sport, etc.). Leur temps quotidien est parcouru d'interactions variées. Nous observons une différenciation des rythmes de l'échange à la fois selon les étudiants et selon les destinataires.

Les échanges sur Facebook dépendent en premier lieu des cercles concernés et du temps consacré, mais peuvent être indépendants du sentiment de proximité ou de l'intimité. La communication avec la famille repose généralement sur d'autres supports (téléphone, Skype) selon un rythme assez espacé 
(hebdomadaire), parfois épisodique pendant l'année scolaire alors que les interactions sont quotidiennes et intenses (sur plusieurs supports) avec des connaissances de la même promotion d'études.

Le rythme dépend ensuite de la nature et de l'objectif des interactions. Certaines relations se conçoivent sur le court terme, mais sont intenses (connaissances de promotion), d'autres relations s'établissent sur le long terme avec des interactions plus ou moins espacées (amis). Toutefois, les interactions sur Facebook peuvent être multi-quotidiennes avec les amis, notamment ceux au Maroc. L'objectif des échanges varie et peut correspondre à des échanges superficiels et de la vie quotidienne (lieu de rendez-vous, informations sur les cours ou les sorties, etc.) ou à des échanges plus intimes et personnels (sentiments, projets, partages, etc.).

La temporalité du rythme des échanges peut également être qualifiée selon l'usage " relationnel " des outils de communication, interrogeant ainsi l'usage multiple de supports de communication. Licoppe et Smoreda (2003) démontrent que l'éloignement engendre souvent une diminution de la fréquence des appels de la famille et des amis proches, mais par contre, il entraîne une augmentation de la durée des appels. Cet usage relationnel se vérifie avec la famille ou les amis proches des étudiants. Les rythmes quotidiens de l'échange sur Facebook s'adaptent aux différentes sphères des étudiants et, au sein de ces sphères, la fréquence des échanges s'établit aussi selon le degré d'intimité.

La présence connectée représente une nouvelle modalité de la sociabilité médiatisée. Maintenir et entretenir l'intimité à distance sont une épreuve du quotidien. Pour les étudiants, Facebook facilite le lien fréquent par la possibilité de communiquer de façon plus large (statut quotidien personnel ou des " amis", messages, tchat, commentaires, etc.). Du point de vue du rythme de l'échange, le web social apporte des changements majeurs. Tout d'abord, le contenu discursif compte moins que l'acte lui-même, c'est-à-dire que l'échange repose sur une attention ou une réaction plus que sur le contenu même, par exemple poster un commentaire sur le statut d'un ami ou "liker " un post. Ensuite, ce moyen de communication n'exige pas de réponse immédiate (excepté pour le " tchat "), le plus significatif étant l'intention personnelle, ce qui rend l'échange particulier. Ainsi, la temporalité des interactions est plus longue que pour les face-à-face ou le téléphone. Enfin, il est parfois difficile de faire la distinction entre les " amis" Facebook et les véritables amis. Les étudiants sont capables d'interagir chaque jour avec un grand nombre d'" amis ", toutefois, les amis les plus proches restent dans le temps et sont la cible d'attentions particulières, plus démonstratives de l'intimité. Les échanges quotidiens par les pratiques de l'immédiat via le réseau social Facebook ont des répercussions sur les temporalités à plus long terme. D'abord, Facebook ajoute de la mémoire, du présentiel ce qui joue dans l'entretien des réseaux. Ensuite, les pratiques de l'immédiat participent de la dynamique sociale au regard du parallèle du temps des pratiques et du temps des réseaux dans la mobilité.

\section{Les étapes de mobilité}

Le web social suit également les temporalités des mobilités. II représente une activité socialisante qui suit les rythmes des rencontres en face à face, mais 
ne les remplace pas (Casilli, 2010). Ainsi, le deuxième niveau de temporalité est celui qui resitue le parcours de mobilité internationale d'une manière plus large, en intégrant les étapes de mobilité, modifiant la représentation du temps et de la projection.

\section{La représentation du temps}

"Aujourd'hui " est composé à la fois des étapes d'hier et des projets de demain. Les étudiants se construisent dans ce rapport au temps, intégrant leur passé au Maroc, leur présent à l'étranger et leurs projets. Le graphe de leur réseau Facebook formalise cette conscience des étapes de leur mobilité. La représentation du temps passe également par les lieux, lesquels émergent sur le graphe. Le temps passé dans certaines villes, dans certains lieux contribue à donner du sens aux lieux. Toutefois, la relation temps/représentation n'est pas toujours proportionnelle. Certains lieux, vécus dans une période plus courte, font davantage sens que d'autres lieux longuement expérimentés. En effet, la ville d'étude à l'étranger occupe généralement une place de premier ordre sur le graphe du fait, notamment, de l'ajout de l'ensemble des connaissances de promotion et de l'effet " étudiant ". L'investissement personnel et social peut dépendre d'une perception du temps cadrée par des échéances connues à l'avance, c'est le cas des étudiants venus en France dans le cadre d'une mobilité pour une durée précise. La représentation du temps et le rapport lieux/relations sociales opèrent de manière différente, comme pour le rendre plus efficace sans pour autant oublier l'investissement social au Maroc, au contraire.

"En fait, Facebook c'est le lien quotidien avec le Maroc. En même temps si on dit le lien, c'est comme si j'étais un peu séparé du Maroc, géographiquement oui, mais je ne me sens pas séparé du Maroc. Il me semble ici que je suis à Marrakech, les gens, le climat. Franchement, il n'y a pas de grande différence entre ici et le Maroc. C'est possible aussi que je me sente un peu chez moi ici, je ne me sens pas étranger. Surtout que je suis venu pour une mission et je connais mes dates " (S., étudiant à Montpellier).

\section{La projection}

La mobilité peut être vécue comme provisoire et renforcer la volonté de s'installer dans le pays d'origine ou, au contraire, avec le temps, le lieu d'origine peut ne plus être reconnu comme avant le départ ; la projection peut donc s'en trouver modifiée. D'une part, les études en mobilité internationale peuvent être vécues comme un chemin initiatique permettant une ouverture d'esprit et une connaissance du monde (tant personnelle que professionnelle) avant de rentrer au Maroc avec ces qualités considérées comme déterminantes pour certains emplois. D'autre part, la mobilité multiplie aussi les choix de vie et joue donc sur les temporalités de l'existence. La prise de conscience des temporalités de la mobilité par la visualisation des graphes des réseaux Facebook incite à questionner le projet migratoire notamment sur la place de la France comme étape ou sur les autres mobilités envisagées. L'entretien des réseaux via Facebook influence la projection des étudiants dans la mesure où la logique de réseau fait écho à une logique d'opportunité où le réseau fait ressource. La mobilité offre la promesse de la découverte d'un nouveau monde, qui reste toujours possible d'élargir. Une première mobilité permet d'en entrevoir d'autres, d'accéder à un autre monde encore inconnu, ou connu par l'intermédiaire des amis, et de s'ouvrir sur un monde plus grand. 


\section{Évolution des réseaux}

Les cercles évoluent selon les temporalités de la mobilité. Les liens de nature différente s'activent, se font et se défont dans le temps. Ici, les liens sociaux sont identifiés pour une population particulière (les étudiants), à un moment précis (les études) et sur un support particulier (Facebook). Même sur cette population jeune, nous voyons apparaître des temporalités de construction des cercles sociaux selon l'évolution des relations.

L'approche par les temporalités commence par l'évènement qui rattache quelqu'un à un cercle : soit l'individu est membre d'un cercle ou a un statut le lui permettant (par exemple l'étudiant marocain pour l'association marocaine des étudiants de Lille), soit son individualité, au-delà de son statut, lui permet d'intégrer un cercle (cercle d'amis, autres associations, sport, intérêts personnels, etc.). Le fait d'être étudiant et marocain confère un statut double permettant I'accès à des cercles à la fois précis et élargis. La combinaison des cercles pour chaque individu rend compte à la fois de ses origines, de sa culture et de son individualité, et ainsi de la complexité et de la richesse de son identité.

Les temporalités permettent également de mettre en avant la dynamique d'évolution des cercles selon le type de relation. Le temps n'est pas le même partout au sein du réseau Facebook. Les temporalités soulignent la stabilité de certains cercles comme la famille, tandis que d'autres, au contraire, ont des formes plus souples, plus élastiques (connaissances de promotion, amis de I'université ou Erasmus, etc.). La stabilité ou l'élasticité des cercles influent sur la structure du réseau dans le temps et son élargissement notamment par le maintien, l'intégration ou l'abandon de certains liens. Un des enjeux est de saisir le passage d'un réseau à un autre dans la mobilité. Il s'agit de comprendre quel réseau est gardé, quel réseau est abandonné, quelles amitiés sont entretenues dans les différentes étapes de la mobilité et comment les réseaux sont mobilisés pour la circulation et la projection. Ces questions s'insèrent dans une problématique plus large de périodisation et de continuité liée aux réseaux sociaux du web. II s'agit notamment d'identifier des frontières et des passerelles entre passé, présent et futur.

\section{Du territoire relationnel au territoire des possibles}

L'approche par les temporalités du web social questionne finalement le rapport à l'espace en termes d'ancrage, de mobilités, de circulations et de l'opérationnalité d'une présence plurielle. De même, l'éclatement ou, au contraire, la concentration du réseau des étudiants laissent entrevoir ou non la possibilité de nouvelles mobilités.

\section{Les contours d'un territoire relationnel en mobilité}

Les temporalités de la mobilité étudiante à partir des réseaux Facebook mettent en exergue la périodisation selon la construction des différentes sphères et renforcent la pertinence d'une approche par la territorialisation mobile et provisoire basée en partie sur les réseaux sociaux du web. En effet, nous pouvons définir un territoire relationnel dont les fondements reposent sur les réseaux sociaux construits et entretenus dans le mouvement. Le territoire révèle 
ses propriétés relationnelles, éphémères, dynamiques.

Les liens sont produits de façon intentionnelle (en acceptant d'être ami sur Facebook). Ils introduisent de nouveaux membres au sein des cercles, agissant ainsi par agglutination et traçant une "frontière en creux " (par absence des autres) (Ma Mung, 2012). Nous pouvons interroger la nature des liens à partir de deux effets distincts : d'un côté le friending ou bridging qui correspond à la construction de nouveaux liens non structurants et, de l'autre, le bonding qui correspond au renforcement des liens existants et à la redondance intra-groupe. II existe un certain équilibre entre bonding (cohésion sociale, liens très soudés) et bridging (connectivité sociale, création de passerelles). Les réseaux Facebook des étudiants font apparaître une multiplexité relationnelle par la coexistence de liens forts et de liens faibles qui interrogent la notion $d^{\prime}$ " ami " du réseau social sur le web. Nous vérifions pourtant que plus le lien est fort, plus les réseaux de relations se superposent. De même pour les liens faibles, il est plus facile de suivre leur actualité au quotidien grâce au réseau du web ; ils sont ainsi certainement plus facilement mobilisables. Cette observation se vérifie surtout pour les groupes Facebook où I'information circule davantage entre les liens faibles réunis. Le réseau social du web permet ainsi des sociabilités à plusieurs échelles. Les liens construits dépendent alors en partie de la nature des cercles évoqués plus haut dans lesquels se trouvent les relations.

À partir du web social, analysé comme un espace de socialisation et un espace de ressources, les résultats amènent à penser le passage au territoire relationnel, en référence également à la sociologie relationnelle de Simmel. Ainsi, le territoire relationnel permet d'appréhender spatialement les relations sociales, de les repositionner au regard du processus de territorialisation, c'està-dire selon les pratiques et les représentations des acteurs.

La territorialisation des étudiants se révèle dynamique puisqu'elle suit les étapes de mobilité et les ancrages qui lui sont associés. Elle met aussi en lien les lieux de la présence plurielle. Un système de mobilité étudiant se construit entre mobilité, migration et circulation en prenant en compte les plots de l'ancrage et le temps des étapes. L'analyse des réseaux sociaux sur le web rend compte d'une nouvelle perception de l'espace par les usages et les pratiques. Le web social offre ainsi un renouvellement de la réflexion sur l'absence/présence et la distance/proximité. L'espace se révèle à travers sa dimension sociale, mais aussi mentale. Évidemment, le contexte contemporain de l'état constant de connexion influe sur cette présence plurielle. La pratique continue du web social par les étudiants participe de cette dynamique. La territorialisation développée par les étudiants marocains en mobilité internationale leur permet une circulation entre les espaces physiques et numériques en leur offrant la possibilité de construire leurs sociabilités selon des réseaux variés et complémentaires. Ainsi, la connexion des étudiants entre plusieurs lieux leur permet d'intégrer cette présence plurielle comme constitutive d'une territorialisation complexe et flexible. La vision de l'espace est alors très fragmentée, fluide et s'apparente à un véritable territoire-réseau puisque celui-ci prend en compte les points de connexion et les flux (ceux produits par les réseaux sociaux) qui les unissent. Cet espace peut s'étirer ou au contraire se rétracter. Ainsi, les territoires construits par les étudiants s'appuient sur des relations sociales élargies dont l'entretien des liens passe en partie par le web. 


\section{Un territoire des possibles: des mobilités potentielles au sein du réseau}

Un territoire des possibles se dessine pour les étudiants en mobilité internationale. Il se définit à partir des relations établies et/ou entretenues à distance et prend sens par une idée de projection envisageable. Il est basé à la fois sur une pré-connaissance des lieux permise par l'expérience des " amis " du réseau et sur une mobilité potentielle, c'est-à-dire non encore réalisée, mais latente et envisageable. Le territoire des possibles résonne comme une liberté de mouvement, mais qui prend sens. Les mobilités ne se déploient pas n'importe où ; les mobilités ont lieu au sein du territoire-réseau. Ainsi, la mobilité reste dynamique, toujours ouverte vers d'autres possibles, prête à englober de nouveaux lieux. Les étudiants ont conscience du caractère provisoire de la mobilité. " Ici " n'est pas une notion exclusive et définitive, mais une option parmi d'autres possibles. Le territoire des possibles rend compte d'expériences projetées, du présent qui se projette vers le futur. Il existe pour n'importe quel individu, mais l'usage du web chez les migrants, et les jeunes particulièrement, augmente le champ des possibles. Les expériences passées et présentes influencent, réorientent voire contraignent le futur et le web social, en tant qu'espace social contracté, permet cette projection. Prenons l'exemple de cet étudiant marocain à Montpellier se projetant dans une autre mobilité internationale pour poursuivre ses études. II fait le choix d'une université aux États-Unis parce qu'il est en contact permanent avec un de ses meilleurs amis du lycée déjà là-bas, avec qui il échange quotidiennement des photos, des informations, lui permettant une pré-connaissance des lieux, des enseignants ou même des évaluations.

L'approche des projections de mobilité des étudiants par le territoire des possibles interroge l'imaginaire migratoire (Simon, 2008). Nous proposons ainsi de le revisiter en élaborant une nouvelle définition de l'ici et de l'ailleurs tenant compte à la fois de l'imaginaire développé par Anderson (2006) et Appadurai (1996) et du rôle des nouvelles technologies, notamment du web social.

L'imaginaire migratoire fait appel au double registre de la dimension individuelle et collective, et à la compétence des migrants à se projeter. II peut ainsi répondre à plusieurs définitions. L'une porte sur la construction du projet de départ par rapport aux conditions de vie des pays d'origine (Simon, 2008 ; Wihtol de Wenden, 2004). Simon positionne l'acte migratoire entre la puissance de l'imaginaire et la demande de réalisation personnelle. L'auteur accorde une importance fondamentale à l'imaginaire migratoire, celui-ci est notamment porté par les nouvelles technologies dont la télévision reste un symbole fort alimentant "le rêve du monde ". Dans cette conception, l'imaginaire migratoire figure comme un moteur de la mobilité, entretenant le rêve d'un ailleurs meilleur et incitant au départ. Les désirs d'ailleurs du migrant avant son départ participent à la construction du projet migratoire. L'autre définition est apportée par Lacroix (2010) et évoque la question du retour et du positionnement vis-à-vis du pays d'origine. L'auteur traite des structures de l'imaginaire migratoire et de la différence entre les nouveaux arrivants et les jeunes nés en France. II définit l'imaginaire migratoire comme "l'ensemble des représentations qui donnent sens à l'acte migratoire et permettent aux acteurs de se positionner dans leurs relations avec les sociétés d'accueil et d'origine "(Lacroix, 2010 : 121). L'auteur questionne l'imaginaire migratoire dans une relation au pays d'origine depuis 
le pays d'accueil, les migrants nourrissant leur imaginaire migratoire par leur propre réflexivité sur leur parcours. Ces deux approches se situent dans un rapport pays d'origine/pays d'accueil, soit dans l'idée du départ, soit dans une projection du retour. De Gourcy (2005) ajoute une dimension de l'imaginaire migratoire par les lieux d'origine vécus notamment à travers l'histoire familiale, sur plusieurs générations. Nous apportons une autre définition de cet imaginaire migratoire reposant sur le lien entre imaginaire, mobilité et technologie. Nous plaçons l'imaginaire migratoire en dehors du simple lien avec le pays d'origine et donc de la seule projection du retour. Notre approche de l'imaginaire migratoire prend en considération les liens entretenus par le web social et les conséquences en termes de mobilité (réellement effectuées ou potentielles) dans d'autres villes et d'autres pays. La pré-connaissance des lieux par l'intermédiaire d'amis favorise l'imaginaire migratoire (partage de photos, de vidéos, etc.). La définition de l'ici et de l'ailleurs évolue dans le sens d'un élargissement de l'accès aux informations relatives à d'autres lieux que ceux connus ou fréquentés et offre donc un élargissement des possibilités de mobilité. Nous sommes face à une population migrante étudiante, qualifiée et intégrée au sein de réseaux sociaux dispersés dans le monde entier. L'imaginaire migratoire se nourrit du partage des expériences des amis. Les représentations sont d'autant plus efficaces avec les échanges instantanés par les réseaux sociaux du web.

\section{Conclusion : le rapport au temps et aux lieux des réseaux Facebook de migrants}

L'article apporte un éclairage sur un type particulier de migration qualifiante, parce qu'éphémère, et insiste sur une appropriation des lieux basée sur les relations sociales et la présence plurielle entre l'ici et le là-bas par l'usage des réseaux sociaux du web. Le web social est un espace social comme l'espace social réel, avec la particularité de contracter l'ensemble des liens et des connaissances acquises au cours des mobilités et des temporalités de la vie. Cet espace social permet donc de cumuler des liens qui peuvent être entretenus, maintenus dans le temps au-delà des pratiques de sociabilités de I'instant présent. Les pratiques du web social facilitent donc le lien avec des personnes proches ou éloignées, géographiquement ou sentimentalement. Par conséquent, le web social facilite le pluri-ancrage en permettant de conserver les liens construits dans des temporalités distinctes, mais dont les pratiques relèvent davantage du temps immédiat. La présence à distance est plus active et entraîne le maintien et le développement des sociabilités, même si le web ne remplace pas les liens directs en face à face. Le web social a opéré un changement radical dans les pratiques des migrants notamment " dans l'activation des réseaux, dans l'organisation et le contrôle des déplacements, sans oublier leur impact sur le vécu de la mobilité " (Diminescu, 2004 : 129). Ainsi, les sociabilités en ligne interrogent sur l'articulation des espaces physiques et du web dans un continuum des relations (Casilli, 2010 : 245). En effet, les interactions médiatisées (sans co-présence physique) impliquent une reformulation des liens dans la distance et de nouvelles représentations de l'espace. Les pratiques du web social annulent ou réduisent les distances de manière éphémère par une mise en relation instantanée des lieux. En parallèle, les pratiques du web social dessinent un territoire des possibles permettant de réinterroger l'imaginaire migratoire. 
Dans la continuité des travaux participant au renouvellement de I'approche des migrations en géographie (Berthomière et Hily, 2006 ; Cortes, 2008 ; Faret, 2003 ; Lacroix, 2005 ; Leclerc, 2011 ; Simon, 2008), notre démarche interdisciplinaire s'est avérée féconde pour se saisir des réseaux sociaux en tant qu'objet de recherche pour la géographie sociale. Sur le plan méthodologique, ce travail a combiné des méthodes et des outils classiques de production de données en géographie sociale avec des méthodes " empruntées " à d'autres disciplines, en introduisant notamment une innovation par les graphes de réseaux et les entretiens de commentaires de ces graphes. En cherchant à spatialiser le réseau, en tant que géographe et ce, à partir du travail de terrain, nous apportons à l'analyse des réseaux de la chair interprétative du point de vue des relations à l'espace. Le réseau n'est plus désincarné. Au-delà des analyses structurales des réseaux en sociologie ou des analyses pointues des réseaux web qui donnent l'impression d'une supra-réalité sociale virtuelle, nous replaçons l'être spatialisé, territorialisé au cœur de la démarche.

Les formes de mise en réseaux des individus, notamment par le web, construisent des territorialités éphémères, mais qui ne sont pas complètement nouvelles. Elles s'articulent à des configurations déjà existantes (ici les filières migratoires) et à des réseaux classiques (familiaux, communautaires). Une nouvelle dynamique se greffe ainsi à de l'ancien, à de l'existant ; qui relativise, en partie, l'idée d'une " révolution " par les réseaux du web.

Le potentiel de compréhension du fonctionnement social des migrants sur le web est vaste et il nous semble que la géographie se doit d'y participer, en ce qu'elle éclaire en particulier le rapport entre le social et le spatial. Les travaux au sein du programme " e-Diasporas " sont, à ce titre, fondateurs. II semble en effet qu'un champ ait été ouvert, exigeant un croisement des disciplines. Par les graphes et les algorithmes associés, les propriétés relationnelles et organisationnelles ouvrent des pistes prometteuses d'analyse des réseaux sociaux. Cela est d'autant plus vrai dans le contexte contemporain des pays arabes où la circulation des qualifiés (et des informations et idées) adopte un visage nouveau au regard des usages du web. Inclure les nouvelles formes de réseaux sociaux, notamment ici Facebook, permet de renforcer la compréhension globale des mobilités étudiantes internationales à travers le monde, mais également de s'intéresser à d'autres catégories de migrants " connectés ". 


\section{Références bibliographiques}

Anderson Benedict (2006) Imagined communities. Reflections on the origin and pread of Nationalism, Londres, Verso, $256 \mathrm{p}$.

Appadurai Arjun (1996) Modernity at Large: Cultural Dimensions of Globalization, Minneapolis, University of Minnesota Press, 224 p.

Berthomière William et Hily Marie-Antoinette (2006) Décrire les migrations internationales. Les expériences de la co-présence, Revue Européenne des Migrations Internationales, (22) 2, pp. 67-82.

Casilli Antonio (2010) Les liaisons numériques : vers une nouvelle sociabilité ?, Paris, Seuil, $331 \mathrm{p}$.

Cortes Geneviève (1998) Migrations, systèmes de mobilité, espaces de vie : à la recherche de modèles, L'espace géographique, 27 (3), pp. 265-275.

De Gourcy Constance (2005) Autonomie dans la migration et dimension mémorielle des lieux, Espaces et sociétés, 4 (122), pp. 187-204.

Di Meo Guy (1998) Géographie sociale et territoires, Paris, Nathan, 320 p.

Diminescu Dana (2012) Diasporas on the Web, Social Science Information, 51 (4), pp. 451-695.

Diminescu Dana (2005) Le migrant connecté. Pour un manifeste épistémologique, Migrations/Société, 17 (102), pp. 275-292.

Diminescu Dana (2004) Le migrant dans un système global des mobilités, in Sylvain Allemand, François Ascher et Jacques Lévy, Les sens du mouvement : modernité et mobilités dans les sociétés urbaines contemporaines, Paris, Belin, pp. 124-129.

Dufoix Stéphane (2003) Les diasporas, Paris, Presses Universitaires de France, $127 \mathrm{p}$.

Faret Laurent (2003) Les territoires de la mobilité : migrations et communautés transnationales entre le Mexique et les États-Unis, Paris, CNRS Editions, 351 p.

Lacroix Thomas (2010) L'imaginaire migratoire. Jeunes Marocains de France, in Françoise Lorcerie Éd., Pratiquer les frontières. Jeunes migrants et descendants de migrants dans l'espace franco-maghrébin, Paris, CNRS Éditions, pp. 121-138.

Lacroix Thomas (2005) Les réseaux marocains du développement. Géographie du transnational et politiques du territorial, Paris, Presses de Sciences Po, 257 p.

Leclerc Éric (2011) Mobilité, spatialité et mondialité. Les informaticiens indiens dans la ville globale, Habilitation à Diriger des Recherches, vol. 4, Université Michel de Montaigne, Bordeaux III, 320 p.

Licoppe Christian et Smoreda Zbigniew (2003) La petite musique du lien : vers une pragmatique de la sociabilité médiatisée, 21 p., [en ligne]. URL : http:// unesco.bell.uqam.ca/article.php?id_article=121

Ma Mung Emmanuel (2012) Enquête exploratoire sur le web des Chinois d'outremer. Morphologie du web et production de la diaspora, e-Diasporas Atlas, 14 p., [en ligne]. URL : http://www.e-diasporas.fr/working-papers/MaMungChinese-FR.pdf 
Marié Michel (1982) Un territoire sans nom, pour une approche des sociétés locales, Paris, Librairie des Méridiens, $176 \mathrm{p}$.

Proulx Serge (2008) Des nomades connectés : vivre ensemble à distance, Hermès, 51, pp. 155-160.

Raffestin Claude (1982) Remarques sur les notions d'espace, de territoire et de territorialité, Espaces et sociétés, 41, pp. 167-171.

Sayad Abdelmalek (1999) La double absence. Des illusions de l'émigré aux souffrances de l'immigré, Paris, Seuil, $437 \mathrm{p}$.

Simmel Georg (1999 [1908]) Le croisement des cercles sociaux, in Georg Simmel, Sociologie. Étude sur les formes de la socialisation, Paris, Presses Universitaires de France, pp. 407-452.

Simon Gildas (2008) La planète migratoire dans la mondialisation, Paris, Armand Colin, 255 p.

Wihtol de Wenden Catherine (2004) La citoyenneté face aux nouveaux visages du migrant, in Sylvain Allemand, François Ascher et Jacques Lévy, Les sens du mouvement : modernité et mobilités dans les sociétés urbaines contemporaines, Paris, Belin, pp. 80-88. 


\section{Sabrina Marchandise}

\section{Le Facebook des étudiants marocains. Territoire relationnel et territoire des possibles}

Cet article questionne la place des pratiques du web dans les réseaux sociaux des étudiants marocains, la structuration et l'organisation de ces réseaux ainsi que la manière dont ils s'inscrivent dans l'espace. L'objectif est de déplacer la problématique de la mobilité internationale des étudiants en faisant le lien entre la dimension migratoire de la mobilité et les nouvelles technologies par une approche des réseaux sociaux de la mobilité. II s'agit de voir en quoi les mobilités contribuent à l'émergence de nouveaux rapports à l'espace et en quoi la spatialisation des réseaux sociaux est révélatrice de ces rapports. À partir des temporalités, l'auteur approche le territoire dans une acception relationnelle, $c^{\prime}$ est-à-dire dont les fondements seraient les relations sociales construites et entretenues dans le mouvement. II discute également le concept de territoire des possibles.

\section{Facebook of Moroccan Students. Relational Territory and Possible Territory}

This article questions the place of web practices in social networks of Moroccan students, the structuring and organization of these networks and how they fit into the space. The objective is to move the issue of international student mobility in linking migration dimension of mobility and new technologies through social networks approach. It comes to see how mobility contribute to the emergence of new relationships to space, and how the spatial distribution of social networks is indicative of these reports. From temporalities, the author approaches the territory in a relational sense, that is to say, the foundations of which would be built and maintained in social relations in the movement. He also discusses the concept of possible territory.

\section{... Facebook de los estudiantes marroquíes. Territorio relacional y territorio de lo posible}

Este artículo interroga la plaza de las prácticas de web en las redes sociales de los estudiantes marroquíes, la estructuración y la organización de estas redes así como la manera en la que se inscriben en el espacio. El objetivo es desplazar la problemática de la movilidad internacional de los estudiantes haciendo el lazo entre la dimensión migratoria de la movilidad y las nuevas tecnologías por un enfoque de las redes sociales de la movilidad. Se trata de ver en qué las movilidades contribuyen a la emergencia de nuevos relaciones al espacio, y en qué la especialización de las redes sociales es revelador de estos relaciones. A partir de las temporalidades, el autor se acerca al territorio en una acepción relacional, es decir cuyos fundamentos serían las relaciones sociales construidas y mantenidas en el movimiento. También analiza el concepto de territorio de lo posible. 